\title{
GPS AlignMent Surveys AND \\ MERIDIAN CONVERGENCE
}

\author{
By Tomás Soler, ${ }^{1}$ Member, ASCE, and Rudolf J. Fury ${ }^{2}$
}

\begin{abstract}
Since the advent of the Global Positioning System (GPS), geodetic azimuths can be accurately computed by simple implementation of well-known 3D concepts. However, when GPS alignment surveys involving azimuths are designed in advance, and later observed and reduced (e.g., during kinematic GPS work), corrections due to the convergence of the meridians should be kept in mind and not ignored. In this study a practical algorithm was used to compare accurately determined "meridian convergence" against the classical formalism available in standard textbooks. The typical approximate formulation available in the open literature was found adequate for GPS engineering surveys such as airport runway profiles, alignment of power lines or conveyor belts, stake positioning in highway construction, etc. A practical GPS survey was used to corroborate the results. Finally, a new $3 \mathrm{D}$ alternative to computing meridian convergence, which is equivalent to the rigorous formalism, is presented.
\end{abstract}

\section{INTRODUCTION} 159):

Meridian convergence can be defined in three ways (Geodetic 1986, p.

- On a curved surface (globular body) it is the mutual approach of the meridians in passing from the equator to the poles. At the equator all meridians are parallel. Extending poleward from the equator, they draw together until they meet at the poles, intersecting in angles equal to their difference of longitude.

- On a curved surface (globular body) and for a given pair of meridians and a given geodesic, it is the difference between the two angles formed by the intersection of the geodesic with the two meridians. Thus the azimuth at one end of the geodesic differs from the azimuth at the other end by $180^{\circ}$ plus or minus the amount of the convergence of the meridians at the endpoints.

- On a map, at a given point, it is the angle measured clockwise from the tangent to the projection of the meridian to the northing coordinate line (grid north) — also called map angle or mapping angle.

The first definition is the most general and the one applicable when Global Positioning System (GPS) methodology is employed. Thus, the geodetic azimuths change between pairs of points $\mathrm{A}$ and $\mathrm{B}, \mathrm{B}$ and $\mathrm{C}, \ldots, \mathrm{F}$ and $\mathrm{G}$, etc., even when all of these points are spanning the same straight line AG. From the above definitions we know that convergence only makes sense between meridians on the surface of the ellipsoid and that the convergence is zero at

${ }^{1}$ Chf., Global Positioning Sys. Branch, Nat. Geodetic Survey, NOS, Nat. Oceanic and Atmospheric Admin., 1315 East-West Hwy., Silver Spring, MD 20910.

${ }^{2}$ Geodesist, Nat. Geodetic Survey, NOS, Nat. Oceanic and Atmospheric Admin., 1315 East-West Hwy., Silver Spring, MD.

Note. Discussion open until January 1, 2001. To extend the closing date one month, a written request must be filed with the ASCE Manager of Journals. The manuscript for this paper was submitted for review and possible publication on June 28, 1999. This paper is part of the Journal of Surveying Engineering, Vol. 126, No. 3, August, 2000. (C)ASCE, ISSN 0733-9453/00/0003-0069-0082/\$8.00 + \$.50 per page. Paper No. 21304. 
the equator and equal to the difference in longitude of the two points if they are close to the poles. The third definition refers to the convergence between the representation of meridians as projected using different mapping equations. This convergence will depend on the particular cartographic projection at hand and will not be discussed further in this paper. However, it can be said that meridian convergence for regular cylindrical projections is zero, and for regular conic and polar azimuthal projections it is a simple function of latitude. Other projections have a more complicated set of equations affected by their intrinsic distortions and are readily available in the geodetic literature. As we will see, the meridian convergence may become several minutes of arc in most surveying applications and thus must not be ignored. It is easily detectable with modern GPS technology and can be significant even when the surveyed points are located at short distances from each other. Despite this fact, the meridian convergence can be approximated to sufficient accuracy by simple equations.

\section{APPROXIMATIONS TO MERIDIAN CONVERGENCE}

\section{Approximation No. 1}

An illustration of the first definition is depicted graphically in Fig. 1(a). The mathematical reasoning followed is the usual treatment of the subject in most classical textbooks [e.g., Zakatov (1962, p. 100)]. The convergence between any two points $\mathrm{A}$ and $\mathrm{C}$ located at the same geodetic latitude $\phi_{\mathrm{A}}$ is visualized by drawing tangents to the ellipsoid along the meridians of $\mathrm{A}$ and $\mathrm{C}$ until they intersect the semiminor axis of the ellipsoid at E. By definition, the angle $d t$ between the tangents is the so-called convergence of the meridians going through points $\mathrm{A}$ and $\mathrm{C}$. The convergence between two arbitrary nearby points A and B, not necessarily on the same parallel [Fig. 1(a)], could

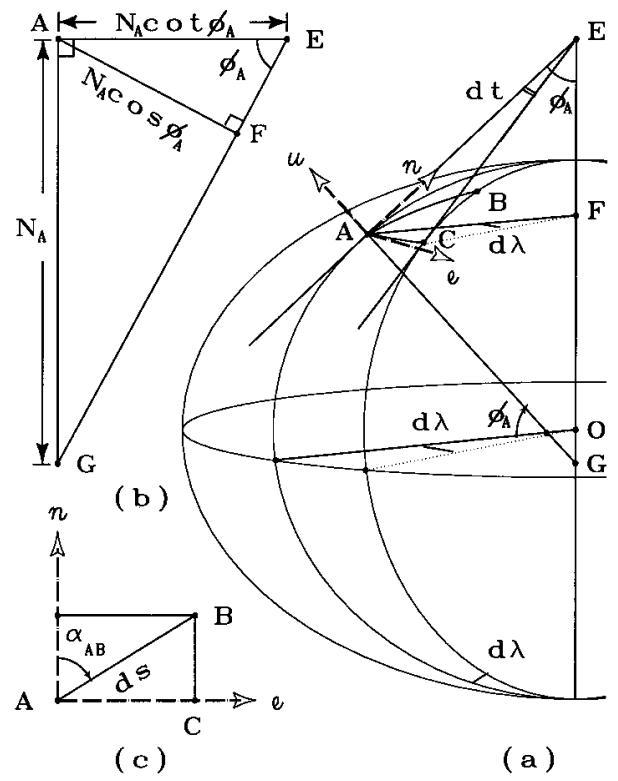

FIG. 1. Meridian Convergence on Surface of Ellipsoid (Diagram Not Drawn to Scale) 
be determined only by assuming a differential local area around point A. Because of this restriction some planar approximations can be enforced. This simplified theory is more than sufficient to cover all usual surveying and surveying-engineering applications. Consequently, the formalism delineated herein will confine the applicability of the equations presented to cases in which the points are located in their local vicinity, not exceeding a distance from point $\mathrm{A}$ of more than $10-\mathrm{km}$ radius. If longer distances are anticipated, the simplifications introduced will no longer be valid, and the second definition should be considered.

A basic but important geometric relationship is

$\operatorname{arc}($ linear units $)=$ central angle $($ radians $) \times$ circular radius (linear units)

Applying the above equality to triangle EAC in Fig. 1(a) and also using Fig. 1(b), we can write

$$
\operatorname{arc} A C=d t N_{\mathrm{A}} \cot \phi_{\mathrm{A}}
$$

Similarly, from triangle FAC we have

$$
\operatorname{arc} A C=d \lambda N_{\mathrm{A}} \cos \phi_{\mathrm{A}}
$$

Because the arcs in (1) and (2) are approximately equal, it immediately follows that

$$
d t=d \lambda \sin \phi_{\mathrm{A}}
$$

For the differential planar case, an element of geodesic $d s$ is equal to an element of normal section and is a straight line [Fig. 1(c)]. From triangle $\mathrm{ABC}$, we obtain

$$
\operatorname{arc} A C=d s \sin \alpha_{\mathrm{AB}}
$$

Equating (4) and (2), an expression for $d \lambda$ is found

$$
d \lambda=d s \sin \alpha_{\mathrm{AB}} /\left(N_{\mathrm{A}} \cos \phi_{\mathrm{A}}\right)
$$

Finally, substituting (5) into (3) provides the formula to compute the convergence of meridians $d t$ as a function of the geodetic latitude $\phi_{\mathrm{A}}$ of point $\mathrm{A}$, the differential distance $d s$ between the two points, and the azimuth $\alpha_{\mathrm{AB}}$ from point $\mathrm{A}$ to point $\mathrm{B}$, namely

$$
d t=d s \sin \alpha_{\mathrm{AB}} \tan \phi_{\mathrm{A}} / N_{\mathrm{A}}
$$

The symbol $N_{\mathrm{A}}$ refers to the principal radius of curvature of the prime vertical of point $\mathrm{A}$, computed as follows: $N_{\mathrm{A}}=a / W$ with $W=\left(1-e^{2} \sin ^{2} \phi_{\mathrm{A}}\right)^{1 / 2}$, where the square of the ellipsoid eccentricity is given in closed form by $e^{2}$ $=2 f-f^{2}$. The ellipsoid of revolution adopted in this investigation is the GRS80 ellipsoid (Moritz 1992); its two geometric parameters of interest are size, defined by the semimajor axis $a=6,378,137 \mathrm{~m}$, and shape, specified by the flattening $f$ or, equivalently, its inverse, $f^{-1}=298.257222101$.

Eq. (6) will be referred to in the text as Approximation No. 1 for determining the meridian convergence.

\section{Approximation No. 2}

An alternative and better approximation to meridian convergence was introduced by the U.S. Coast and Geodetic Survey (Formulas 1933, pp. 8 and 97). The final equation is given as 


$$
d t=d \lambda \sin 1 / 2\left(\phi_{m}\right) \sec 1 / 2\left(\phi_{\mathrm{B}}-\phi_{\mathrm{A}}\right)+(d \lambda)^{3} \sin \phi_{m} \cos ^{2} \phi_{m} / 12
$$

where $\phi_{m}=1 / 2\left(\phi_{\mathrm{A}}+\phi_{\mathrm{B}}\right)=$ mean latitude of points $\mathrm{A}$ and $\mathrm{B}$. The value of $d \lambda$ required in the above equation was previously given in (5).

\section{RIGOROUS MERIDIAN CONVERGENCE}

\section{Geodetic Azimuth $\boldsymbol{\alpha}_{\mathrm{AB}}$}

Now, attention will be paid to the unambiguous definition of azimuth $\alpha_{\mathrm{AB}}$. To clarify the symbolism, two similar, but distinct, definitions are reviewed:

- Geodesic azimuth $\alpha_{\mathrm{AB}}^{\prime}$ - the azimuth of the geodesic between two points $\mathrm{A}$ and $\mathrm{B}$ on the surface of the reference ellipsoid measured at A.

- Geodetic azimuth $\alpha_{\mathrm{AB}}$ - the azimuth between two spatial points A and $\mathrm{B}$ (not necessarily on the ellipsoid) measured clockwise from the geodetic north on the geodetic horizon plane of $\mathrm{A}$, this plane being at a right angle to the ellipsoidal normal. This parameter is referred to by some authors as 3D azimuth (Burkholder 1997).

When points $\mathrm{A}$ and $\mathrm{B}$ are near each other $(\leq 10 \mathrm{~km}), \alpha_{\mathrm{AB}} \sim \alpha_{\mathrm{AB}}^{\prime}$. Consequently, a unique notation $\alpha_{\mathrm{AB}}$ and the definition of geodetic azimuth will be assumed throughout the remainder of this work. It is well known that geodetic azimuths can be related to astronomic azimuths (a physical quantity) through the components of the deflection of the vertical and the so-called Laplace's equation or condition (Heiskanen and Moritz 1967, pp. 186 and 190). A combination of GPS and geodetic leveling observations also permits the accurate determination of vertical deflections (Soler et al. 1989).

Geodetic azimuths between any two arbitrary points are the only ones that can be determined by 3D GPS techniques and methods and thus are the only ones of interest in current geodetic and surveying GPS practice. However, the definition of geodetic azimuth makes more sense if the two points are intervisible, this being independent of the distance between such a pair of points. The same interpretation does not apply to geodesic azimuths that are strictly referred to points lying on the surface of the ellipsoid. Hence, a geodetic azimuth between points on opposite sides of the reference ellipsoid, although computable, does not usually have practical interest. However, determining instantaneous geodetic azimuths of visible celestial objects or artificial satellites is perfectly sound and sometimes necessary. A practical example related to communication geostationary satellites was presented in Soler and Eisemann (1994).

Recalling Fig. 1(a), notice that we have drawn the local geodetic frame [i.e., $(e, n, u)$ ] at point A. Local geodetic frames are commonly referred to in the geodetic literature as east-north-up frames (Soler and Hothem 1988). The particular notation used here is consistent with right-handed coordinate frames, the only ones considered throughout this paper.

The typical data output in a GPS survey contains, among other quantities, the components of vectors $\left(\Delta x_{\mathrm{AB}}, \Delta y_{\mathrm{AB}}, \Delta z_{\mathrm{AB}}\right)$ between points (e.g., A and $\mathrm{B})$. However, these components are given with respect to a local $(x, y, z)$ conventional terrestrial frame. The $(x, y, z)$ frame is parallel to the geocentric conventional terrestrial reference frame $(\mathbf{x}, \mathbf{y}, \mathbf{z})$ (see Appendix II for definitions). The conversion between local conventional terrestrial frames $(x, y$, $z)$ and local geodetic frames $(e, n, u)$ is accomplished by applying a transformation rotation matrix $[R]$, which enforces parallelism between those frames. 
The geodetic azimuth $\alpha_{\mathrm{AB}}$ and "geodetic altitude" $\nu_{\mathrm{AB}}$ (i.e., geodetic vertical angle) between two points $\mathrm{A}$ and $\mathrm{B}$ can be computed using the following two equations:

$$
\begin{gathered}
\tan \alpha_{\mathrm{AB}}=e_{\mathrm{AB}} / n_{\mathrm{AB}} \\
\tan v_{\mathrm{AB}}=u_{\mathrm{AB}} /\left(e_{\mathrm{AB}}^{2}+n_{\mathrm{AB}}^{2}\right)^{1 / 2}
\end{gathered}
$$

This implies that the components of the vector $\mathbf{A B}\left(e_{\mathrm{AB}}, n_{\mathrm{AB}}, u_{\mathrm{AB}}\right)$ must be known along the local geodetic coordinate frame $(e, n, u)$. These can be calculated by transforming the components $\left(\Delta x_{\mathrm{AB}}, \Delta y_{\mathrm{AB}}, \Delta z_{\mathrm{AB}}\right)$ along the $(x$, $y, z)$ frame at $\mathrm{A}$ as given by GPS, into the $(e, n, u)$ frame. This transformation is performed by using the rotation matrix $[R]_{\mathrm{A}}$ defined as follows [e.g., Soler (1976) and Soler and Hothem (1988)]:

$$
\begin{gathered}
{[R]_{\mathrm{A}}=R_{1}\left(1 / 2 \pi-\phi_{\mathrm{A}}\right) R_{3}\left(\lambda_{\mathrm{A}}+1 / 2 \pi\right)=R_{3}(1 / 2 \pi) R_{2}\left(1 / 2 \pi-\phi_{\mathrm{A}}\right) R_{3}\left(\lambda_{\mathrm{A}}\right)} \\
\quad=\left[\begin{array}{ccc}
-\sin \lambda & \cos \lambda & 0 \\
-\sin \phi \cos \lambda & -\sin \phi \sin \lambda & \cos \phi \\
\cos \phi \cos \lambda & \cos \phi \sin \lambda & \sin \phi
\end{array}\right]_{\mathrm{A}}
\end{gathered}
$$

and the matrix equation

$$
\left\{\begin{array}{l}
e_{\mathrm{AB}} \\
n_{\mathrm{AB}} \\
u_{\mathrm{AB}}
\end{array}\right\}=[R]_{\mathrm{A}}\left\{\begin{array}{l}
\Delta x_{\mathrm{AB}} \\
\Delta y_{\mathrm{AB}} \\
\Delta z_{\mathrm{AB}}
\end{array}\right\}
$$

If the components $\left(\Delta x_{\mathrm{AB}}, \Delta y_{\mathrm{AB}}, \Delta z_{\mathrm{AB}}\right)$ are not immediately known, they can be determined from the geocentric Cartesian coordinates of points A and B

$$
\left\{\begin{array}{l}
\Delta x_{\mathrm{AB}} \\
\Delta y_{\mathrm{AB}} \\
\Delta z_{\mathrm{AB}}
\end{array}\right\}=\left\{\begin{array}{l}
\mathbf{x}_{\mathrm{B}} \\
\mathbf{y}_{\mathrm{B}} \\
\mathbf{z}_{\mathrm{B}}
\end{array}\right\}-\left\{\begin{array}{l}
\mathbf{x}_{\mathrm{A}} \\
\mathbf{y}_{\mathrm{A}} \\
\mathbf{z}_{\mathrm{A}}
\end{array}\right\}
$$

The rectangular coordinates, $\mathbf{x}, \mathbf{y}, \mathbf{z}$ of any arbitrary point can also be computed from their known curvilinear geodetic coordinates $(\lambda, \phi, h)$ employing well-known expressions

$$
\left\{\begin{array}{l}
\mathbf{x} \\
\mathbf{y} \\
\mathbf{z}
\end{array}\right\}=\left\{\begin{array}{c}
(N+h) \cos \phi \cos \lambda \\
(N+h) \cos \phi \sin \lambda \\
{\left[N\left(1-e^{2}\right)+h\right] \sin \phi}
\end{array}\right\}
$$

where $N$, as mentioned above, is the principal radius of curvature in the prime vertical. A noniterative inverse transformation of (13) to compute geodetic coordinates from Cartesian coordinates based on the work of Bowring (1985) was published in Soler and Hothem (1989).

\section{Numerical Investigation}

To ascertain the accuracy of (6) and (7) when used as an alternative for the approximation of meridian convergence, a numerical simulation was performed. It is based on a rigorous 3D approach that emulates the logic applied in everyday GPS work.

A horizontal line joining two arbitrary stations A and B separated by a distance of $10 \mathrm{~km}$ was selected to restrict the outcome of the analysis to practical engineering surveys. Because the expected results are azimuth dependent, the values of $\alpha_{\mathrm{AB}}$ were increased by increments of $15^{\circ}$ from the meridian of point A. Similarly, to account for the dependence of convergence 


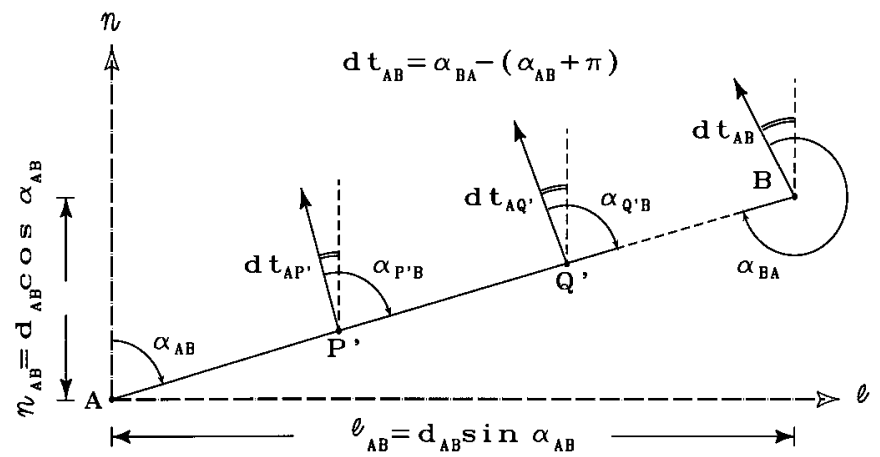

FIG. 2. Meridian Convergence Along Line AB of Azimuth $\alpha_{A B}$ (Diagram Not Drawn to Scale)

$$
\begin{aligned}
& \begin{array}{ccc}
\left(\alpha_{\mathrm{AB}}, v_{\mathrm{AB}}=0, \mathrm{~d}_{\mathrm{AB}}=10 \mathrm{~km}\right) & \left(\lambda_{\mathrm{A}}, \phi_{\mathrm{A}}, \mathrm{h}_{\mathrm{A}}=0\right) & \left(\alpha_{\mathrm{AB}}, v_{\mathrm{AP}}=0, \mathrm{~d}_{\mathrm{AP}}=1 \mathrm{~km}\right) \\
\downarrow & \downarrow & \downarrow \\
\left(e_{\mathrm{AB}}, n_{\mathrm{AB}}, u_{\mathrm{AB}}=0\right) & \downarrow & \left(e_{\mathrm{AP}}, n_{\mathrm{AP}}, u_{\mathrm{AP}}=0\right) \\
\downarrow & \downarrow & \downarrow \\
\left(\Delta x_{\mathrm{AB}}, \Delta y_{\mathrm{AB}}, \Delta z_{\mathrm{AB}}\right) & \left(x_{\mathrm{A}}, y_{\mathrm{A}}, z_{\mathrm{A}}\right) & \left(\Delta x_{\mathrm{AP}}, \Delta y_{\mathrm{AP}}, \Delta z_{\mathrm{AP}}\right)
\end{array} \\
& \left(x_{\mathrm{B}}, y_{\mathrm{B}}, z_{\mathrm{B}}\right) \quad \underbrace{}_{\left(x_{\mathrm{P}}, y_{\mathrm{P}}, z_{\mathrm{P}}\right)} \\
& : \quad\left(\lambda_{\mathrm{p}}, \phi_{\mathrm{p}}, \mathbf{h}_{\mathrm{p}}\right) \\
& : \quad\left(\lambda_{\mathrm{P}^{\prime}}=\lambda_{\mathrm{P}}, \phi_{\mathrm{P}^{\prime}}=\phi_{\mathrm{p}}, \mathrm{h}_{\mathrm{P}^{\prime}}=0\right) \\
& (x_{\mathrm{B}}, \underbrace{\left.y_{\mathrm{B}}, z_{\mathrm{B}}\right)} \quad\left(x_{\mathrm{p},}, y_{\mathrm{p}^{\prime}}, z_{\mathrm{p}^{\prime}}\right) \\
& \left(\Delta x_{P^{\prime} \mathrm{B}}, \Delta y_{\mathrm{P}^{\prime} \mathrm{B}}, \Delta z_{\mathrm{P}^{\prime B} \mathrm{~B}}\right) \\
& \perp \\
& \left(e_{\mathrm{P}^{\prime} \mathrm{B}}, n_{\mathrm{P}^{\prime} \mathrm{B}}, \boldsymbol{u}_{\mathrm{P}^{\prime} \mathrm{B}}\right) \\
& \left(\alpha_{P^{\prime} B}, v_{P^{\prime} B}, d_{P^{\prime} B}\right) \\
& \alpha_{\mathrm{P}^{\prime} \mathrm{B}}-\alpha_{\mathrm{AB}}=\mathrm{dt}_{\mathrm{P} \mathrm{B}} \\
& \begin{array}{c}
\left(\alpha_{\mathrm{AB}}, v_{\mathrm{AB}}=0, \mathrm{~d}_{\mathrm{AB}}=10 \mathrm{~km}\right) \\
\vdots \\
\vdots \\
:
\end{array} \\
& \begin{array}{cc}
\left(\lambda_{\mathbf{P}^{\prime}}, \phi_{\mathbf{P}^{\prime}}, \mathrm{h}_{\mathrm{P}^{\prime}}=0\right) & \left(\alpha_{\mathrm{P}^{\prime} \mathrm{B}}, v_{\mathrm{P}^{\prime} \mathrm{B}}=0, \mathrm{~d}_{\mathrm{P}^{\prime} \mathrm{Q}}=1 \mathrm{~km}\right) \\
\vdots & \downarrow \\
\vdots & \vdots
\end{array}
\end{aligned}
$$

FIG. 3. Algorithm to Rigorously Determine Meridian Convergence

on distance, intermediate points between $\mathrm{A}$ and $\mathrm{B}$ were introduced having a constant spacing of $1 \mathrm{~km}$ between them. Recall that from (6) the convergence $d t$ is also a function of the latitude of the initial point $\mathrm{A}$ where the original azimuth $\alpha_{\mathrm{AB}}$ is measured; thus, to identify exactly the two points between which the convergence is measured, a more explicit notation $d t_{\mathrm{AB}}$, indicating the convergence at $\mathrm{B}$ between the meridians of $\mathrm{A}$ and $\mathrm{B}$, is introduced (Fig. 2). Similarly, we write $d t_{\mathrm{AP}^{\prime}}, d t_{\mathrm{AQ}^{\prime}}$, etc. The test performed in this numerical investigation was based on an a priori selection of geodetic coordinates for point A, specifically, $\lambda_{\mathrm{A}}=100^{\circ} \mathrm{W}, \phi_{\mathrm{A}}=40^{\circ} \mathrm{N} ; h_{\mathrm{A}}=0$, which places $\mathrm{A}$ on the reference ellipsoid near the center of the conterminous United States.

The flowchart of the rigorous algorithm used to compute the convergence of meridians between two points $\mathrm{A}$ and $\mathrm{B}^{\prime}$ on the ellipsoid and its graphical 


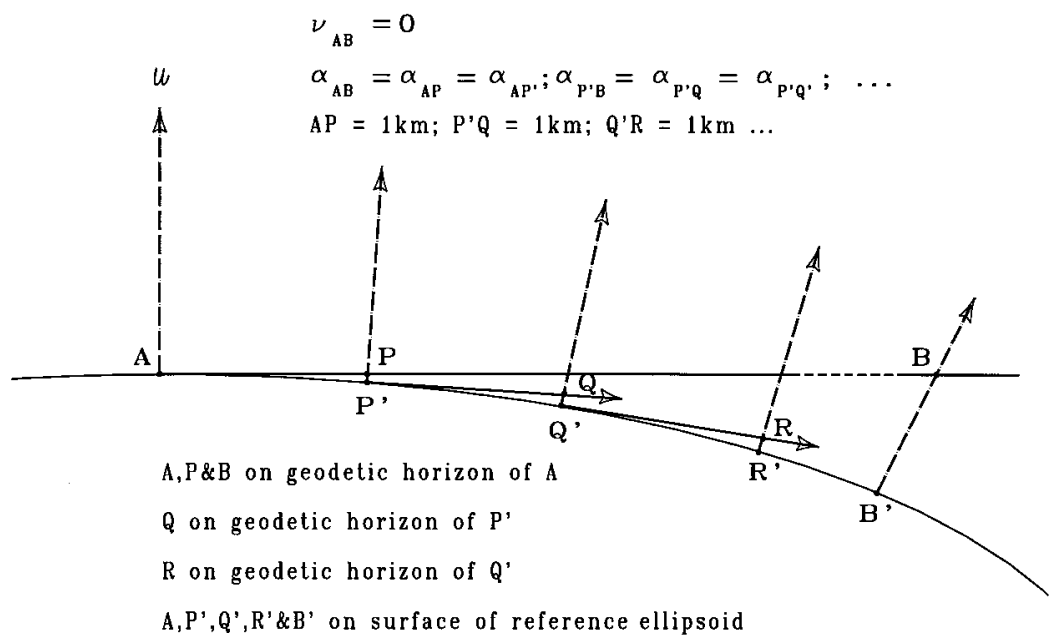

FIG. 4. Schematic Diagram to Rigorously Compute Meridian Convergence

interpretation is recounted step-by-step in Figs. 3 and 4. Notice that $\mathrm{P}^{\prime}$ is the projection of $\mathrm{P}$ on the surface of the ellipsoid $\left(h_{\mathrm{P}^{\prime}}=0\right)$. Thus all the computations are performed along tangents to the surface of the ellipsoid at points $\mathrm{A}, \mathrm{P}^{\prime}, \mathrm{Q}^{\prime}$, etc. It should be remembered that the normals to the ellipsoid at $\mathrm{P}^{\prime}, \mathrm{Q}^{\prime}, \mathrm{R}^{\prime}$, etc., are not generally contained on the same plane. Exceptions include points along the same meridian or at the equator where the convergence is zero. Thus, the azimuths (e.g., $\alpha_{\mathrm{AB}}$ and $\alpha_{\mathrm{P}^{\prime} \mathrm{Q}}$ ) are not measured on the same local geodetic plane. However, because the distances AP, $\mathrm{P}^{\prime} \mathrm{Q}$, etc. (Fig. 4) are only $1 \mathrm{~km}$, the error introduced is negligible. This approach is more rigorous than computing the meridian convergence using the equality

$$
d t_{\mathrm{AB}}=\alpha_{\mathrm{BA}}-\left(\alpha_{\mathrm{AB}}+\pi\right)
$$

because the distance between $\mathrm{A}$ and $\mathrm{B}$ is $10 \mathrm{~km}$ and the local geodetic planes at $\mathrm{A}$ and $\mathrm{B}$ are not parallel. Our simulation shows that although the ellipsoidal height of point $\mathrm{B}$ is $7.8 \mathrm{~m}$ (distance $\mathrm{BB}^{\prime}$ in Fig. 4) the difference in convergence computed using the rigorous algorithm and (14) is only $0^{\prime \prime} .02$. Thus for all practical purposes, once the coordinates of $\mathrm{P}^{\prime}$ are known (Fig. 2), the meridian convergency $d t_{\mathrm{AP}^{\prime}}$ can be determined using (14).

The rigorous meridian convergence as a function of distance and azimuth between two arbitrary points with the restrictions mentioned above is shown in Fig. 5. The convergence of the meridians is significant even at short distances (e.g., 100 arc seconds at $5 \mathrm{~km}$ and azimuth of $45^{\circ}$ ) and, if neglected, can easily accumulate with distance. The convergence between meridians increases with the value of azimuth, reaching a maximum at azimuths of $90^{\circ}$. This could be explained by simple geometric principles. Restricting the study to the first quadrant, and assuming a constant distance from point $\mathrm{A}$, the separation between the meridians of the two points, which is directly proportional to the convergence, increases with azimuth. Fig. 5 is strictly valid only for points of $\phi=40^{\circ}$. Considering that the parallels increase in curvature per unit length approaching the north pole, the meridians get closer and closer for a constant interval of longitude, Fig. 6 shows the value of the meridian convergence for a constant azimuth $\alpha=45^{\circ}$ at various latitudes.

It should be emphasized that the magnitude of the convergence, although 


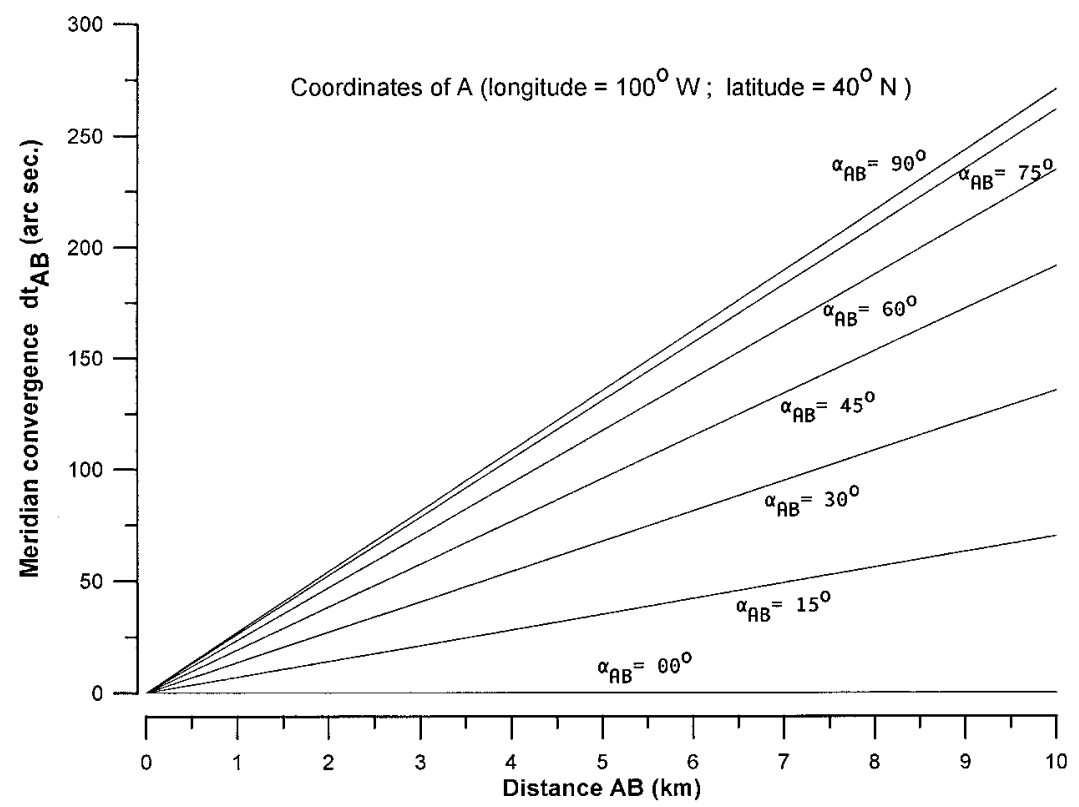

FIG. 5. Rigorous Meridian Convergence as Function of Distance and Azimuth

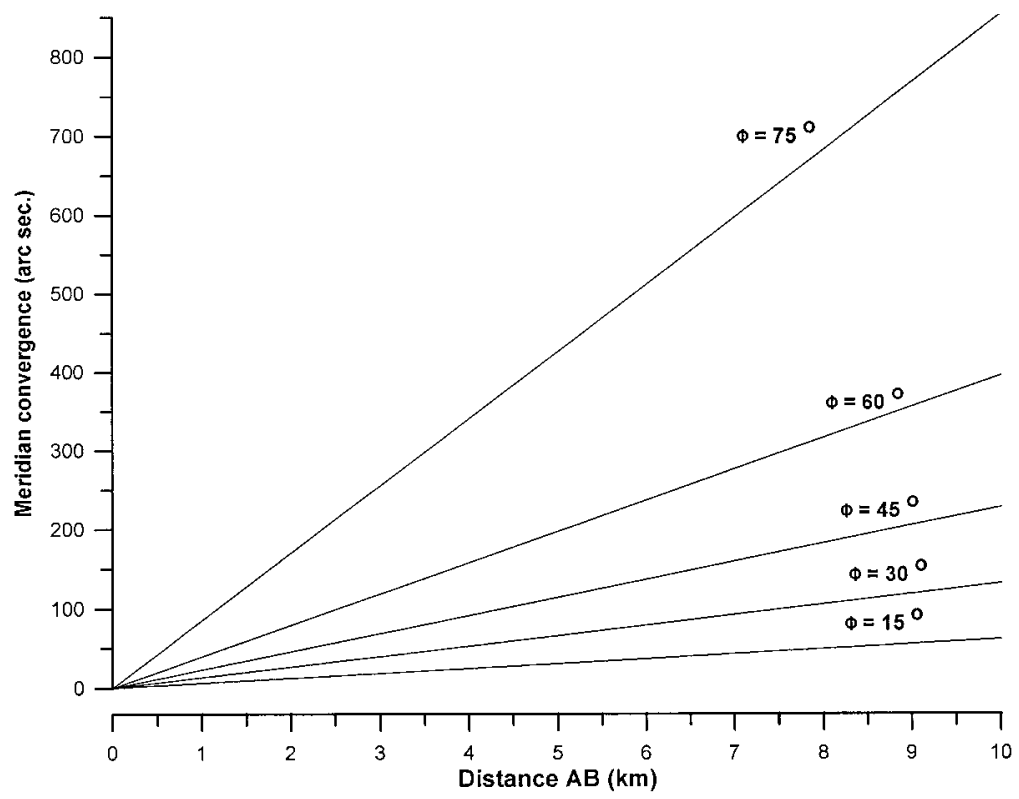

FIG. 6. Meridian Convergence at Different Latitudes $\phi$ and Constant Azimuth $\alpha=45^{\circ}$ (Constant)

76 / JOURNAL OF SURVEYING ENGINEERING / AUGUST 2000 


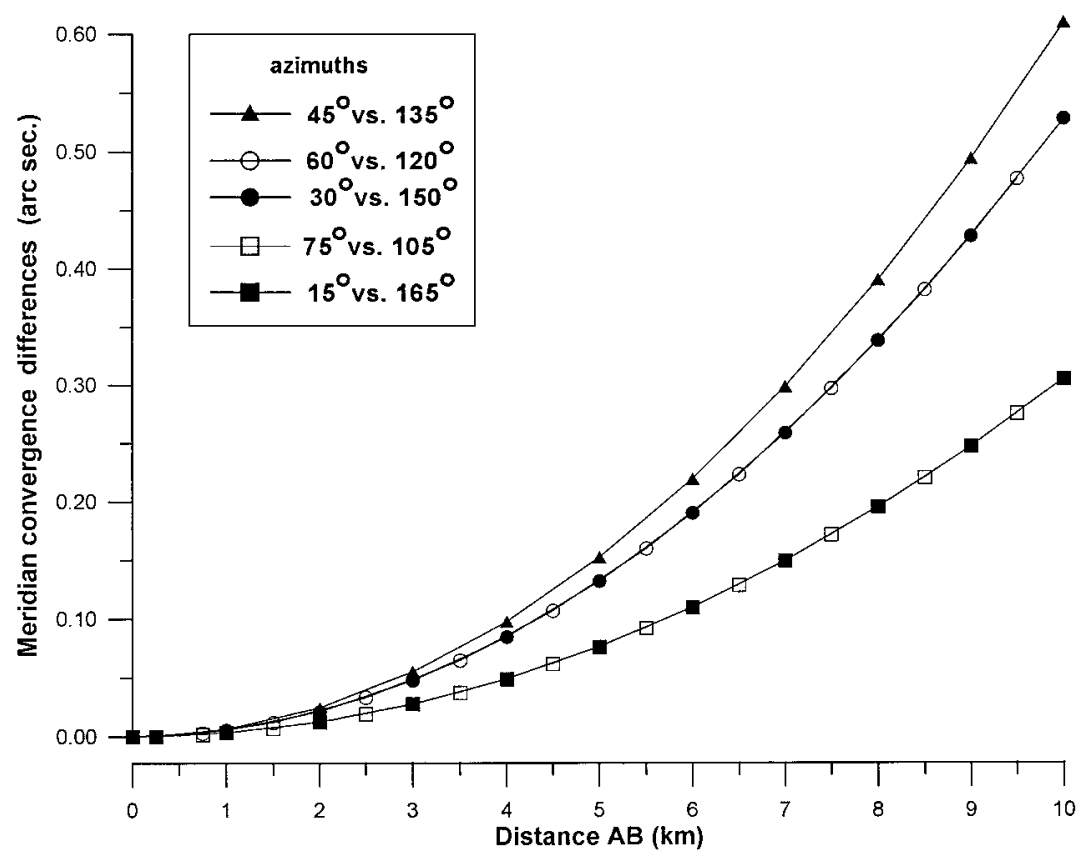

FIG. 7. Difference of Meridian Convergence between Symmetric Northern and Southern Points with Respect to $90^{\circ}$ Azimuth

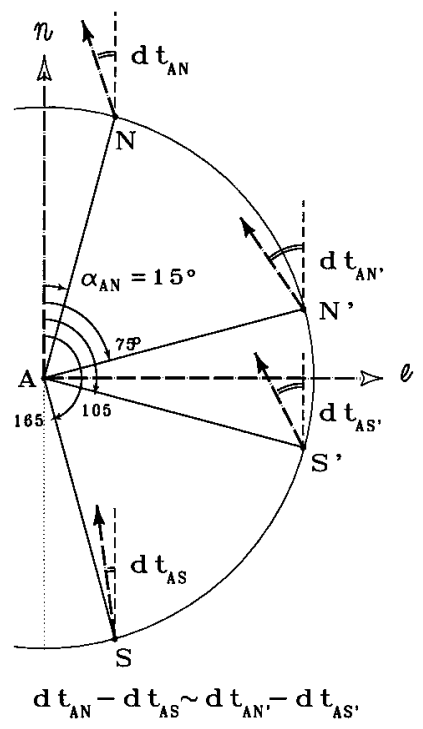

FIG. 8. Graphic Display of Meridian Convergence at Northern and Southern Points (Diagram Not Drawn to Scale) 


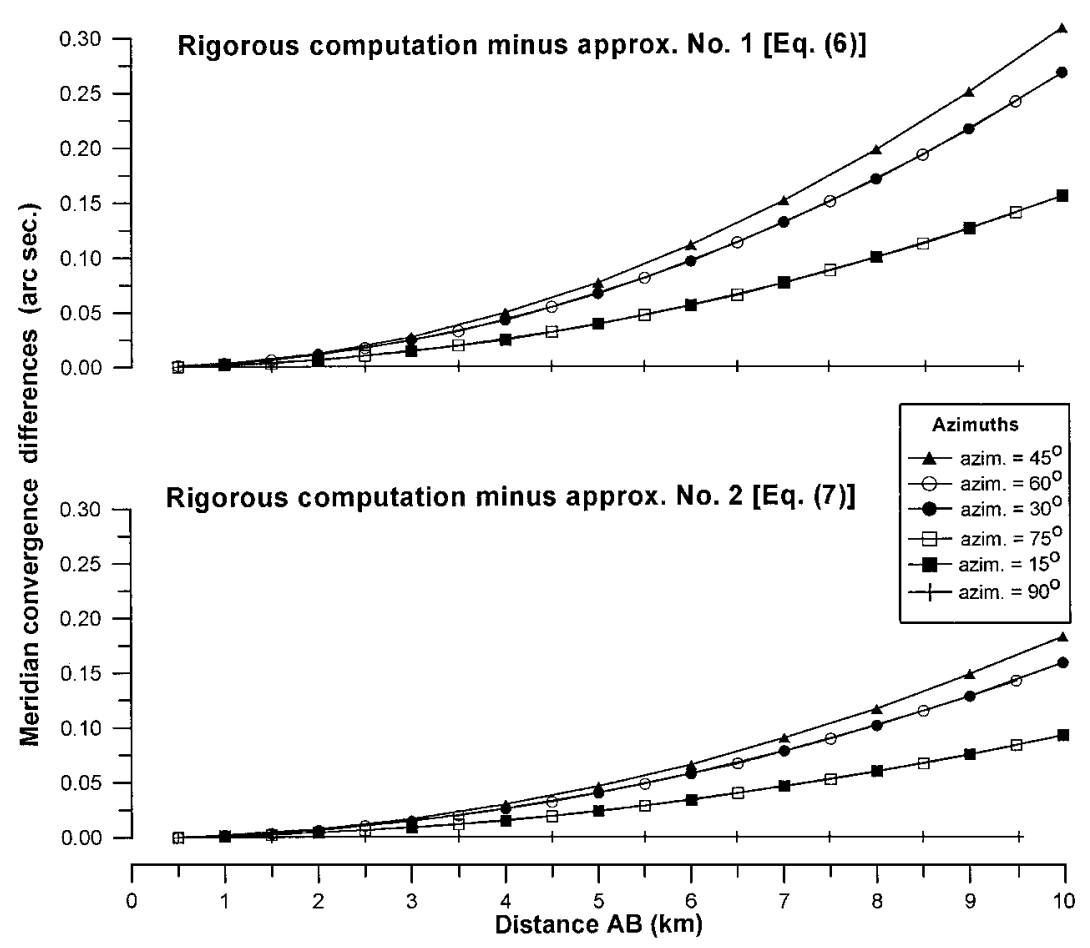

FIG. 9. Differences in Meridian Convergence (Rigorous-Approximate)

symmetric with respect to longitude due to the rotational symmetry of the ellipsoid, is not likewise symmetric with respect to latitude on account of the changing north-south curvature of the ellipsoid. Fig. 7 depicts the differences in meridian convergence for points symmetrically located with respect to the east-west line of point A. The maximum difference in convergence between northern and southern points happens at azimuths of $45^{\circ}$ and $135^{\circ}$ and amounts to about only 0.15 arc seconds at distances of $5 \mathrm{~km}$. A conclusion not obvious beforehand can be drawn. Because of the symmetry implicit in the geometry of the problem, the differences between the northern and southern values of the convergence are almost identical for the pairs $15^{\circ}$ versus $165^{\circ}$ and $75^{\circ}$ versus $105^{\circ}$, and $30^{\circ}$ versus $150^{\circ}$ and $60^{\circ}$ versus $120^{\circ}$. Fig. 8 is a graphical interpretation of this interesting finding, which displays two cases of the example studied.

When the approximate equations [(6) and (7)] were compared to the rigorous approach, the differences encountered up to distances of $10 \mathrm{~km}$ are not significant. This is depicted in Fig. 9, which shows that the discrepancy between the rigorous algorithm and the two approximate equations is maximum at $45^{\circ}$ azimuths. Refined (7) gives slightly better results. The graph shows that, at $45^{\circ}$ azimuths and distances of $10 \mathrm{~km}$, the maximum difference between the rigorous algorithm and (6) reaches only 0.30 arc seconds. The actual convergence at this distance and azimuth is 180 arc seconds (Fig. 5). This implies that, in the worst possible scenario, we are making only a $0.2 \%$ error when we approximate the convergence of meridians by (6). For distances $<6 \mathrm{~km}$, the difference between the approximate equation and the rig- 


$$
\begin{aligned}
& \overrightarrow{\mathrm{AA}}^{\prime \prime}=2(\overrightarrow{\mathrm{AB}}) \\
& \alpha_{\mathrm{A}^{\prime} \mathrm{B}}=\alpha_{\mathrm{AB}} \\
& \mathrm{dt}_{\mathrm{AB}}=\alpha_{\mathrm{BA}^{\prime \prime}}-\alpha_{\mathrm{AB}}
\end{aligned}
$$

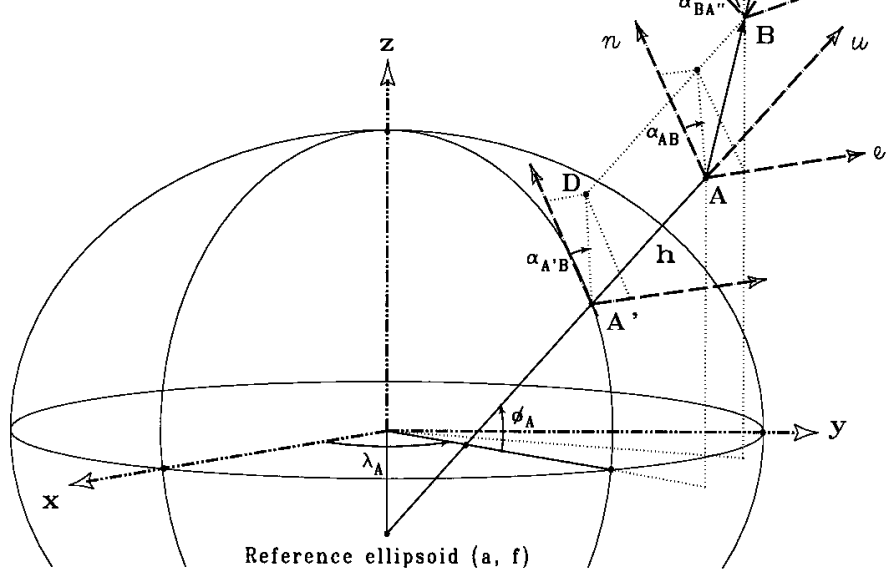

FIG. 10. General 3D Meridian Convergence (Diagram Not Drawn to Scale)

orous numerical approach does not exceed 0.1 arc seconds. This difference is smaller than the precision of most theodolites used in surveying engineering field work. Another characteristic of Fig. 9 shows that the differences between the rigorous algorithm and (6) and (7) are symmetric with respect to $45^{\circ}$ azimuths and zero at $90^{\circ}$ azimuths. These are consequences of the assumptions implicit in the simplifications of the approximate equations. For example, for short distances and azimuths of $90^{\circ}$ the three approaches give the same answer for the value of the meridian convergence. In Fig. 1(a), arc AC will approximate a straight line, and the three approaches to compute meridian convergence are equivalent.

It is important to recognize that in surveying practice it is incorrect to assume that the azimuth from $\mathrm{P}^{\prime}$ to $\mathrm{B}$ (Fig. 2) with $\mathrm{P}^{\prime}$ on the line $\mathrm{AB}$ has the same azimuth $\alpha_{\mathrm{AB}}$ as the line $\mathrm{AB}$ itself. The convergence of meridians is significant even for distances typically involved in precise surveying alignments and should never be ignored.

Once the computations on the ellipsoid were implemented according to the logic described, a more general 3D approach was investigated. Fig. 10 shows that by the definition of geodetic azimuth between two points $\mathrm{A}$ and $\mathrm{B}$, the azimuth $\alpha_{\mathrm{AB}}$ at $\mathrm{A}$ is equal to the azimuth $\alpha_{\mathrm{A}^{\prime} \mathrm{B}}$ from $\mathrm{A}^{\prime}$ (the projection of $\mathrm{A}$ on the surface of the ellipsoid) to B measured on the geodetic horizon plane of $\mathrm{A}^{\prime}$. This results from the definition of local coordinate systems $(e, n, u)$, which are parallel at $\mathrm{A}$ and $\mathrm{A}^{\prime}$. Thus, the intersection of the plane ABDA' with the local geodetic horizon planes of $\mathrm{A}$ and $\mathrm{A}^{\prime}$ produces the same geodetic azimuths, in other words, $\alpha_{\mathrm{AB}}=\alpha_{\mathrm{A}^{\prime} \mathrm{B}}$. What then is the convergence $d t_{\mathrm{AB}}$ ? By definition it is the spatial angle between the $n$-axis at $\mathrm{B}$ and an axis parallel to the $n$-axis of A at point B (Fig. 10).

Based on the above definition, the convergence $d t_{\mathrm{AB}}$ can be approximately written as follows: 


$$
\begin{aligned}
& \longleftarrow \text { Eq.(11) } \longrightarrow \\
& \leftarrow \text { Eq. (8) } \rightarrow
\end{aligned}
$$

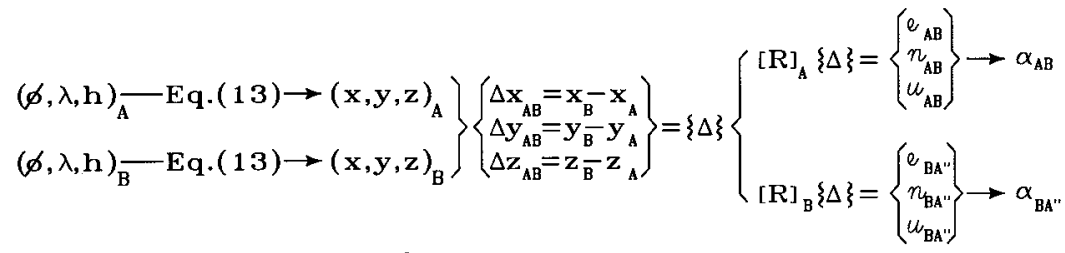

$$
\begin{aligned}
& \mathbf{d t}_{\mathrm{AB}}=\alpha_{\mathrm{BA}}-\alpha_{\mathrm{AB}} \\
& \mathrm{dt}_{\mathrm{AB}}>0\left\{\begin{array}{l}
\phi_{\mathrm{A}}>0 ; \quad 0<\alpha_{\mathrm{AB}}<180 \\
\phi_{\mathrm{A}}<0 ; \quad 180<\alpha_{\mathrm{AB}}<360
\end{array}\right. \\
& \mathrm{dt}_{\mathrm{AB}}<0\left\{\begin{array}{l}
\phi_{\mathrm{A}}>0 ; 180<\alpha_{\mathrm{AB}}<360 \\
\phi_{\mathrm{A}}<0 ; \quad 0<\alpha_{A B}<180
\end{array}\right.
\end{aligned}
$$

FIG. 11. Algorithm to Compute 3D Meridian Convergence

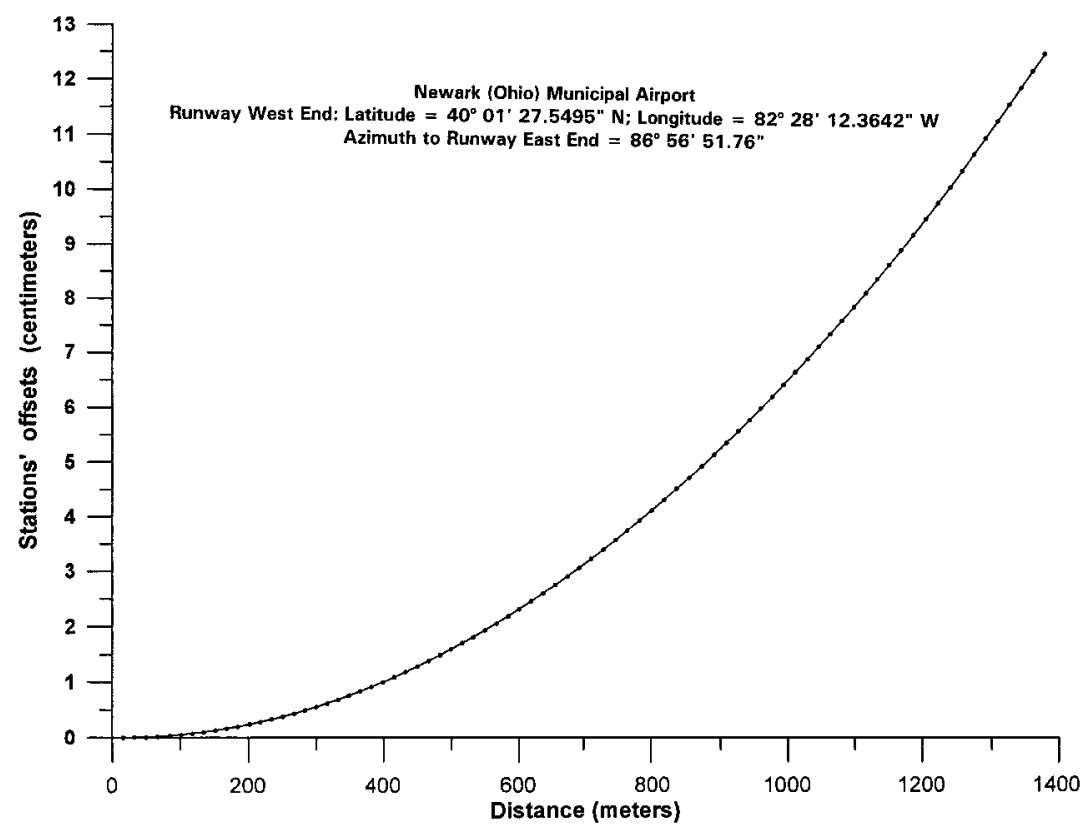

FIG. 12. Circular Cumulative Station Offsets due to Neglecting Meridian Convergence Errors

$$
d t_{\mathrm{AB}}=\alpha_{\mathrm{BA}^{\prime \prime}}-\alpha_{\mathrm{AB}}
$$

where the point $\mathrm{A}^{\prime \prime}$ is the tip of a vector $\mathbf{B} \mathbf{A}^{\prime \prime}$ that has the same orientation and magnitude as the vector $\mathbf{A B}$. The approximation involved is the assumption that the axis through $\mathrm{B}$ parallel to the $n$-axes at $\mathrm{A}$ is in the local geodetic horizon of $\mathrm{B}$. The algorithm used to compute the convergency anywhere in 
space is presented in Fig. 11. We restricted the computations to points that are $<10 \mathrm{~km}$ apart, but the same logic can be expanded to longer distances if necessary. Restricting ourselves to distances of a maximum of $10 \mathrm{~km}$, the difference between the rigorous ellipsoidal and 3D algorithms only amounted to $0^{\prime \prime} .007$ when $v_{\mathrm{AB}}=5^{\circ}$. Because of its generality and geometric simplicity, the $3 \mathrm{D}$ approach to compute meridian convergence is recommended in practice.

\section{FIELD EXAMPLE}

To appreciate the practical implications of neglecting the meridian convergence, a real example will be discussed. The test area selected is located at the Municipal Airport of Newark, Ohio. The two ends of a typical runway, points $\mathrm{A}$ and $\mathrm{B}$, are accurately surveyed using static GPS procedures and ties to nearby points of the National Continuously Operating Reference Stations (CORS) network. Then, a kinematic GPS survey is performed starting at runway endpoint $\mathrm{A}$, reaching $\mathrm{B}$, and returning to $\mathrm{A}$ to provide redundancy and to check for blunders. The principal objective of the survey is to have an accurate geodetic height profile between points $\mathrm{A}$ and $\mathrm{B}$ along the line defining the center of the runway. An interpolation routine was developed to determine the average height of the two measurements along the line $\mathrm{AB}$ at intervals of $50 \mathrm{~m}$. The locations of the equidistant points along route $\mathrm{AB}$ were computed using the coordinates of the initial point $\mathrm{A}$ and the azimuth from A to B, which could be accurately computed from the coordinates of A and B. Not correcting this azimuth for the effects of meridian convergence (the rigorous algorithm was used) at each 50-m interval results in the accumulation of circular positional errors depicted in Fig. 12. When performing surveying alignments of distances even as short as 1,400 $\mathrm{m}$ - the actual length of this particular runway - and for an azimuth close to $90^{\circ}$ a closing error of about $13 \mathrm{~cm}$ (almost $1 / 2 \mathrm{ft}$ ) would be accumulated by not correcting for meridian convergence at each 50 -m section.

\section{CONCLUDING REMARKS}

The correction for meridian convergence is critical when computing geodetic coordinates of points that should be accurately aligned between two known stations $\mathrm{A}$ and $\mathrm{B}$ by traversing intermediate points $\mathrm{P}^{\prime}, \mathrm{Q}^{\prime}$, etc. As Fig. 5 shows, meridian convergence depends on the location of the initial point $\mathrm{A}$, the geodetic azimuth $\alpha_{\mathrm{AB}}$, and the distance between $\mathrm{A}$ and $\mathrm{B}$. Thus, (6) or (7) are used to approximate the meridian convergence when computing coordinates for planning alignment projects. If preferred, a new more rigorous $3 \mathrm{D}$ algorithm could be implemented to determine the value of the meridian convergence between any two points in space, although its practicality is restricted to topographic mapping applications. It is important to realize that meridian convergence errors can be detected using modern GPS techniques. Neglecting this correction may introduce inadmissible errors when calculating the coordinates of points along profiles that must be positioned in the field using traversing GPS methods. Hence, meridian convergence becomes significant for distances exceeding $1 \mathrm{~km}$ and should be taken into account when rigorous GPS surveying alignments are established.

\section{ACKNOWLEDGMENTS}

The writers appreciate the contribution of their National Geodetic Survey colleague Jeff Olsen, whose inquiry prompted this investigation. The writers are also indebted to an anonymous reviewer for helpful comments. 


\section{APPENDIX I. REFERENCES}

Bowring, B. R. (1985). "The accuracy of geodetic latitude and height equations." Survey Rev., 28(October), 202-206.

Burkholder, E. F. (1997). "Three-dimensional azimuth of a GPS vector." J. Surv. Engrg., ASCE, 123(4), 139-146.

Formulas and tables for the computation of geodetic positions. (1933). U.S. Coast and Geodetic Survey Spec. Publ. No. 8. U.S. Government Printing Office, Washington, D.C.

Geodetic glossary. (1986). National Geodetic Survey, Silver Spring, Md.

Heiskanen, W. A., and Moritz, H. (1967). Physical geodesy, W. H. Freeman and Co., San Francisco; Reprinted (1981), Inst. of Phys. Geodesy, Technical University, Graz, Austria.

Moritz, H. (1992). “Geodetic reference system 1980.” Bull. Géodésique, 66(2), 187192.

Soler, T. (1976). "On differential transformations between Cartesian and curvilinear (geodetic) coordinates." Rep. No. 236, Dept. of Geod. Sci., Ohio State University, Columbus, Ohio.

Soler, T., Carlson, A. E., Jr., and Evans, A. G. (1989). "Determination of vertical deflections using the global positioning system and geodetic leveling." Geophys. Res. Letter, 16(7), 695-698.

Soler, T., and Eisemann, D. W. (1994). "Determination of look angles to geostationary communication satellites." J. Surv. Engrg., ASCE, 120(3), 115-127.

Soler, T., and Hothem, L. D. (1988). "Coordinate systems used in geodesy: Basic definitions and concepts." J. Surv. Engrg., ASCE, 114(2), 84-97.

Soler, T., and Hothem, L. D. (1989). "Important parameters used in geodetic transformations." J. Surv. Engrg., ASCE, 115(4), 414-417.

Zakatov, P. S. (1962). A course in higher geodesy, National Technical Information Service, U.S. Department of Commerce, Washington, D.C. (translated from Russian).

\section{APPENDIX II. NOTATION}

The following symbols are used in this paper:

$(e, n, u)=$ local (right-handed) geodetic coordinate system at any point $(\lambda, \varphi, h) ; e$-axis points to (geodetic) east, $n$ to (geodetic) north, and $u$ to (geodetic) zenith;

$h=$ geodetic height (i.e., height above ellipsoid);

$N=$ principal radius of curvature in prime vertical plane;

$(x, y, z)=$ local (terrestrial) frame; origin is at point of observation and $x-, y$-, and $z$-axes are parallel to $\mathbf{x}-, \mathbf{y}_{-}$, and $\mathbf{z}$-axes, respectively;

$(\mathbf{x}, \mathbf{y}, \mathbf{z})=$ conventional terrestrial reference frame; Earth's fixed geocentric coordinate system; $\mathbf{z}$ points toward conventional terrestrial pole, $\mathbf{x}$ passes through point of zero longitude as defined by International Earth Rotation Service; $\mathbf{y}$ forms right-handed coordinate system with $\mathbf{x}$ and $\mathbf{y}$ [e.g., ITRF97, epoch 1997.0; WGS84 (G873), epoch 1997.0];

$\alpha=$ geodetic azimuth;

$\alpha^{\prime}=$ geodesic azimuth; and

$\left(\lambda_{\mathrm{A}}, \phi_{\mathrm{A}}, h_{\mathrm{A}}\right)=$ curvilinear geodetic coordinates (longitude, latitude, and ellipsoid height) of an arbitrary point A. 